\title{
Performance Evaluation of Energy-Efficient Cluster based Algorithms in Wireless Sensor Network
}

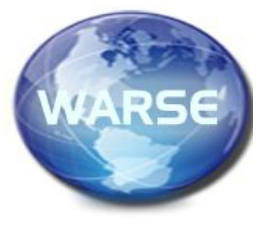

\author{
Harshita Chaurasiya ${ }^{1}$ Dr. Shivnath Ghosh ${ }^{2}$ \\ M.Tech Scholar ${ }^{1}$ \\ Dept of CSE, MPCT, Gwalior \\ harshitachaurasiya27@gmail.com \\ Associate Professor ${ }^{2}$ \\ Dept of CSE, MPCT, Gwalior \\ shivnath.cs@gmail.com
}

\begin{abstract}
The wireless sensor network consists of small power sources with a limited number of nodes with a limited number of communication, calculation and archiving functions. It is also impossible to change stacks of sensor nodes because of their large-scale use in harsh environments. Therefore, the longevity of the network is the main concern of the WSN. This article analyzes a comparative score for three different cluster-based routing protocols in WSN, such as the LEACH, TEEN, and SEP routing protocols. The number of sensor nodes in WSN is large and only one node in the resource is incredibly limited. The main goal of routing protocol design is to improve energy efficiency and extend network life. MATLAB Network Simulation Tool is used to simulate the routing protocol and data analysis.
\end{abstract}

Keywords -Wireless Sensor Network, Clusterbased Routing Protocol, LEACH Protocol, SEP Protocol, TEEN Protocol, Energy Efficiency

\section{INTRODUCTION}

Wireless sensor networks (WSN) comprise of several sensor nodes deployed randomly in a field having limited memory, processing capacity and power mounted on one board which cannot be recharged or replaced as shown in Fig. 1. Therefore, to extend the network lifetime is the major challenge in sensor networks. Recently many researchers have implemented many routing techniques with one common challenge, i.e., save the energy to enhance the life of the network.

In WSN, one of the major challenges is to save the energy or to enhance the efficiency. To achieve this, paradigm is shifted from a single-level hierarchy to multi-level hierarchy and clustering is the best way to implement multi-level hierarchy in the network. A clustering technique forms 2-level hierarchy. In level 1 , some member nodes close to each other form a group called clusters. In cluster, all the member nodes sense the data and forward to the one particular node called Cluster Head (CH). In level 2, cluster heads aggregates the data forward to the Base Station (BS). BS can be located within the field or at another place; [1]. The base station can be a sink node or a personal computer equipped with better power supply which is used for data storage. The major concern of clustering technique is a selection of cluster head. Clustering is an NP-hard problem to minimize the total energy dissipation in a network. For the $\mathrm{N}$ number of sensor nodes in WSN, there are $2 n-1$ different combination to elect the sensor node as $\mathrm{CH}$ or non-CH in each solution for the WSN [2].

LEACH [6] is an important clustering scheme that uses a stochastic approach to select CHs. Ensures longevity of the network compared to direct communication between the node and the base station. However, Leach suffers from the following disadvantages: (i) It takes neither the residual energy of the nodes nor its distance from BS during the electoral process $\mathrm{CH}$ and (ii) assumes that the $\mathrm{CH}$ distribution in the network is uniform. The problem of the LEACH protocol has been superseded by many protocols, taking into account the unused energy of the nodes during the electoral process.

Sharma and Sharma [8] proposed a modified LEACH protocol called the LEACH EEE protocol. The new version of the LEACH protocol recognizes a multilevel clustering approach to minimize the communication distance between the nodes and the $\mathrm{CHMs}$ proposed with $\mathrm{CH}$. The simulations were performed in MATLAB and the results showed that EEE LEACH was more energy efficient than the LEACH protocol. Christian and Soni [9] introduced LEACH with the popular cluster-based structures in WSN. This work used the improved LEACH 
protocol (ILEACH) and compared it with the LEACH protocol. In terms of FND (first node matrices) and HND (half of the node matrices), the duration sensors were evaluated to ensure consistency and performance efficiency in WSN. Mehta et al. [10] developed a LEACH (C-LEACH) system with equalized clusters to initialize and maintain uniform sized clusters to locate them on the network. From the point of view of the energy constraint, this algorithm required a minimum routing overhead to extend the traditional LEACH generation time. A concept of adoption was also integrated for the nodes of the orphan cluster that can be effectively integrated into adjacent clusters. El and Shaaban [11] designed a modified LEACH protection (MS-Leach), data security and $\mathrm{CH}$ node authentication, using coupled keys shared between the CHs. The investigation has shown that it has effective safety features and has achieved the WSN safety objectives. Regarding several existing security-specific protocols, the MS-LEACH version seems to dominate with their safe, obviously effective properties. $\mathrm{Xu}$ et al. [12] LEACH improvement proposal (E-LEACH) to improve the LEACH hierarchical routing protocol. In this case, the $\mathrm{CH}$ was selected randomly and defined in lap time. To balance the network load, it took into account the residual power at the sensor node and the changes in lap time depended on the size of the cluster. The results of the simulation have shown that this protocol has increased the duration of the network by $40 \%$ compared to the LEACH algorithm.

Zhang et al. [13] presented a weighted energy weighting scheme based on a time prediction factor (FABR EBRM), in which the adjacent node is chosen based on the mass and the compactness of the connection. forward energy. Furthermore, a natural reconstruction project for the localized topology was designed. In the FABR EBRM research was compared with Leach and EEUC, the simulation results showed that the FABR EBRM and the Leach EEUC exceeded to compensate for energy consumption, prolong service life and ensure the quality of the service (QoS)) in WSN .

Geetha et al. [14] suggested optimizing many routing protocols to maximize the efficiency of resource constraints in WSN. Clustering algorithms are gaining importance as they increase the WSN lifespan through $\mathrm{CH}$ channel selection and data aggregation approach. LEACH as the initial cluster routing protocol proved to be better than other algorithms. This work was supported by two major
Leach and Leach-C (centralized) clustering protocols, against the NS2 tools for different conditions and the simulation results for the performance indicators were against the latency and duration of the network. This work was completed by commenting on the compliance with the simulated results for these protocols. Gambhir and Fatima [15] provided a version of the LEACH protocol called Optimized LEACH (OP-LEACH) to reduce energy intake in the WSN. Both the current LEACH and the LEACH operational project were evaluated using simulations using the OMNeT ++ simulator, which demonstrated that Op-Leach worked better on the LEACH protocol.

Ankit Thakkar [16] presented the Advanced LEACH protocol on distance and energy called DEAL. DEAL considers the energy and the suppression of a node during the electoral process of the parent company. The results of the simulation show that DEAL extends the stability period and reduces the period of instability compared to the ALEACH protocol.

\section{LEACH PROTOCOL}

WSN is a subset of ad hoc networks. WSN consists of autonomous sensors distributed specifically for the cooperative monitoring of physical or environmental conditions such as temperature, sound, vibration, pressure, movement, etc. This is essential in the cluster routing protocol. Routing strategies and security issues are a major challenge for research. Today, WSN has proposed a number of routing protocols, but the most well-known protocols are hierarchical protocols such as LEACH. Hierarchical protocols are defined to reduce energy consumption by aggregating data and reducing transmissions to the base station [2].

The Leach protocol is a TDMA-based MAC protocol. The main goal of this protocol is to improve the life of wireless sensor networks by reducing energy. The Leach protocol consists of two phases:

- Set-up phase

- Steady phase

The operation of the leaching protocol consists of several shifts with two phases per turn. The Leach protocol is a typical representation of the hierarchical routing protocol. It is self-regulated and selforganized [2]. The leach protocol uses a cycle as a unit, each cycle consisting of a cluster configuration phase and a stationary memory to reduce unnecessary energy costs. 


\section{A. Set-up phase}

In the set-up phase, the main objective is to create clusters and select the cluster head for each cluster by selecting the sensor node with maximum energy [3]. Set-up phase has three fundamental steps:

- Cluster head advertisement

- Cluster set up

- Creation of transmission schedule

In the first step, the cluster manager sends the publication package to inform the cluster nodes that they have become a cluster header based on the following formula:

$$
T(n)=\frac{p}{1-p\left[\operatorname{rmod}\left(\frac{1}{\rho}\right)\right]}
$$

$\mathrm{T}$ (n) is the threshold. Where ' $\mathrm{p}$ ' is the number of nodes in the cluster head ' $\mathrm{r}$ ' is the current number of rounds.

The node becomes the cluster head for the current turn if the number is less than the T (n) threshold. Once the node has been selected as the cluster head, it can not become the cluster manager again until all nodes in the cluster become cluster headers. This is useful for balancing energy consumption.

In the second step, non-clustered header nodes receive the cluster header post and then send a join request to the cluster header to indicate that they belong to the cluster under this header. cluster. All nodes outside the cluster save a lot of energy by constantly switching off their transmitters and turning them on only when they have to send something to the head of the cluster [2].

In the third step, each cluster manager selected creates a transfer plan for the member nodes of your cluster. The TDMA schedule is created based on the number of nodes in the cluster. Each node then sends its data in the assigned schedule [3].

\section{B. Steady phase}

In the stable phase, cluster nodes send their data to the cluster head. The sensor members of each cluster can communicate with the cluster head only with a single jump transfer. The cluster header groups all the collected data and transmits the data to the base station, either directly or through another cluster head, along with the static route defined in the source code. After a specified time interval, the network returns to set-up phase.

\section{TEEN PROTOCOL}

TEEN (network protocol with threshold-sensitive energy efficiency sensor) is aimed at reactive networks and, to our knowledge, is the first protocol developed for reactive networks. In this scheme, the cluster header sends to its members, at each cluster time change, in addition to the attributes.

Hard threshold $\left(\mathrm{H}_{\mathrm{T}}\right)$ : this is a threshold for the perceived attribute. This is the absolute value of the attribute beyond which the node that collects this value must activate the issuer and report it to its cluster leader.

Soft Threshold $\left(\mathrm{S}_{\mathrm{T}}\right)$ : this is a small change in the value of the perceived attribute that determines the activation of the node and the sending of its transmitter.

The knots continually hear their environment. When a parameter of the attribute set reaches its strict threshold for the first time, the node activates its transmitter and sends the acquired data. The measured value is stored in an internal variable of the node called measured value $\left(\mathrm{S}_{\mathrm{V}}\right)$. The nodes will then pass data in the current cluster period if both of the following conditions are true:

1. The current value of the sensed attribute is greater than the hard threshold.

2. The current value of the sensed attribute differs from $S_{\mathrm{V}}$ by an amount equal to or greater than the soft threshold.

Whenever a node transmits data, $S_{\mathrm{V}}$ is equal to the current value of the detected attribute. Therefore, the strict threshold attempts to reduce the number of transfers by allowing the node to send only if the detected attribute is in the region of interest. The soft threshold also reduces the number of transfers by eliminating any transfer that would occur if there was little or no change in the perceived attribute once the strict threshold has been reached.

\section{SEP PROTOCOL}

The SEP protocol ensures that each node knows the total energy of the network and previously calculates the optimal probability of the cluster head based on the remaining energy of the node [9]. Initially, each node is assigned a weight corresponding to the optimal probability $\mathrm{P}_{\text {opt }}$. The weight must be equal to the initial energy of each node divided by the initial energy of the common node [10]. It also defines the $\mathrm{P}_{\mathrm{nrm}}$ probability of the weighted vote for the common nodes, the $\mathrm{P}_{\mathrm{adv}}$ probability of the weighted vote for the extended nodes.

$$
P_{n r m}=\frac{P_{o p t}}{1+a m}
$$




$$
P_{a d v}=\frac{P_{o p t}}{1-a m}(1+a)
$$

Where $\mathrm{a}=$ Initial energy of the advanced node and a multiple of the initial energy of the ordinary node.

$\mathrm{M}=$ The proportion of the advanced node in the total number of nodes. Common nodes and advanced nodes become clusters head and $\mathrm{T}$ threshold respectively.

$$
\begin{gathered}
T=\frac{P_{n r m}}{1-P_{n r m}\left[\operatorname{rmod}\left(\frac{1}{P_{n r m}}\right)\right]} \\
T=\frac{P_{a d v}}{1-P_{a d v}\left[\operatorname{rmod}\left(\frac{1}{P_{a d v}}\right)\right]}
\end{gathered}
$$

Where $\mathrm{r}=$ The current number of rounds consequently, the nodes with high residual energy can turn out to be the nodes with lower residual energy.

\section{COMPARISON OF DIFFERENT CLUSTERING PROTOCOLS}

In this research work comparison of LEACH, SEP and TEEN was done by using MATLAB simulation tool. The nodes are randomly distributed in the area of $100 \times 100 \mathrm{~m}^{2}$. The base station is located at the center point $(50,50)$. The simulation parameters are given below in table I.

Table I: Simulation Parameters

\begin{tabular}{|c|c|}
\hline Parameter Name & Values \\
\hline Network Area & $100 * 100$ \\
\hline Number of nodes & 200 \\
\hline Packet Size & $4000 \mathrm{bits}$ \\
\hline Initial Energy, E $\mathrm{O}$ & $.5 \mathrm{~J}$ \\
\hline Transmitter Energy, ETX & $50 \mathrm{~nJ} / \mathrm{bit}$ \\
\hline Receiver Energy, ERX & $50 \mathrm{~nJ} / \mathrm{bit}$ \\
\hline $\begin{array}{c}\text { Amplification Energy for } \\
\text { short distance, Efs }\end{array}$ & $10 \mathrm{pJ} / \mathrm{bit} / \mathrm{m}^{2}$ \\
\hline $\begin{array}{c}\text { Amplification Energy for } \\
\text { long distance, Emp }\end{array}$ & $0.0013 \mathrm{pJ} / \mathrm{bit}^{2} \mathrm{~m}^{2}$ \\
\hline Number of Rounds & 200 \\
\hline
\end{tabular}

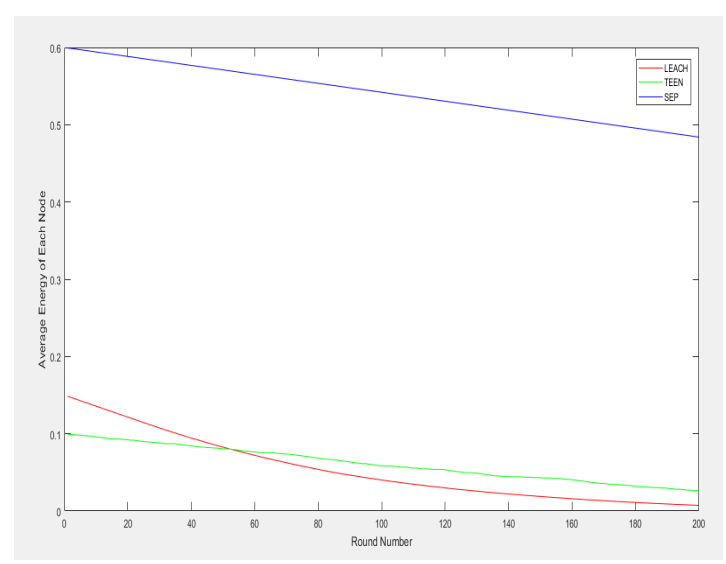

Figure 1: Average Energy Evaluation in LEACH, SEP and TEEN Protocols

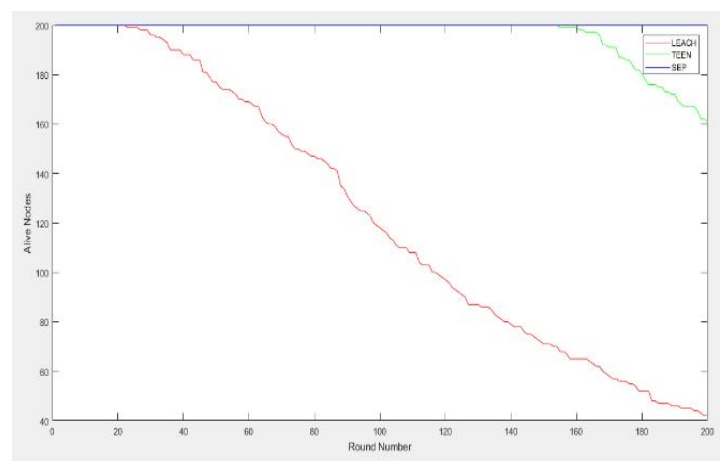

Figure 1: Alive Node Evaluation in LEACH, SEP and TEEN Protocols

\section{CONCLUSION}

In this paper, a comparative performance is evaluated among two different clustering techniques such as LEACH and SEP. Compared with the LEACH, TEEN and SEP has the following benefits:

- The initial energy of different nodes is different and the SEP protocol selects the energy of the cluster head to lengthen the time of death of the first node which is to extend the period of stability.

- The throughput of the SEP protocol is higher than that of other clustering protocols.

- The average energy lost by the SEP protocol is quite less as compared to LEACH and TEEN.

- Number of dead nodes is more in LEACH protocol as well as in TEEN with respect to SEP protocol.

So, keeping these result analysis future work will be focused on designing an algorithm that would be more energy efficient with respect to SEP clustering algorithm. In future work, cluster head selection would also be focused for more efficient results. 


\section{REFERENCES}

[1] Akyildiz, I. F., Su, W., \& Sankarasubramaniam, Y., "A survey on sensor networks", IEEE Communications Magazine, 40(8), 102-114, 2002. https://doi.org/10.1109/MCOM.2002.1024422

[2] Yuan, Y., Yang, Z., He, Z., \& He, J., "An integrated energy aware wireless transmission system for QoS provisioning in wireless sensor network", Computer Communications, 29, 162$172,2006$. https://doi.org/10.1016/j.comcom.2005.05.015

[3] Akyildiz, I. F., Melodia, T., \& Chowdhury, K. R., "A survey on wireless multimedia sensor Networks", Computer Networks, 51, 921-960, 2007. https://doi.org/10.1016/j.comnet.2006.10.002

[4] Zhu, Y., Wu, W., Pan, J., \& Tang, Y., "An energy-efficient data gathering algorithm to prolong lifetime of wireless sensor networks", Computer Communications. doi:10.1016/j.comcom.2009.11.008, 2009. https://doi.org/10.1016/j.comcom.2009.11.008

[5] Alka Singh, Shubhangi Rathkanthiwar, Sandeep Kakde, "LEACH Based- Energy Efficient Routing Protocol for Wireless Sensor Networks", IEEE, 2016.

[6] koge, R., \& Chugh, A., "Various Aspects of LEACH in Wireless Sensor Network: A Survey", International Journal Online of Science, Vol 2, issue 9, 2016. Available at: http://ijoscience.com/ojsscience/index.php/ojssci ence/article/view/111.

[7] . Sharma, M., Shaw, A. K.: Transmission time and throughput analysis of EEE LEACH, LEACH and direct transmission protocol: a simulation based approach. Advanced Computing, 3(6), 75. (2012).

[8] Sharma. M., Sharma. K.: An energy efficient extended LEACH (EEE LEACH). In Communication Systems and Network Technologies (CSNT), 2012 International Conference on (pp. 377-382). IEEE. (2012, May).

[9] Christian. A., Soni. H.: Lifetime prolonging in LEACH protocol for wireless sensor networks. In Intelligent Systems and Signal Processing (ISSP), 2013 International Conference on (pp. 350-355). IEEE. (2013, March). https://doi.org/10.1109/ISSP.2013.6526933

[10] Mehta. R., Pandey. A., Kapadia. P.: Reforming clusters using $\mathrm{C}$-LEACH in wireless sensor networks. In Computer Communication and Informatics (ICCCI), 2012 International Conference on (pp. 1-4). IEEE. (2012, January). https://doi.org/10.1109/ICCCI.2012.6158875

[11]El. M., Shaaban. E.: Enhancing S-LEACH security for wireless sensor networks. In Electro/Information Technology (EIT), 2012
IEEE International Conference on (pp. 1-6). IEEE. (2012, May).

[12]Xu. J., Jin. N., Lou. X., Peng. T., Zhou, Q., Chen. Y.: Improvement of LEACH protocol for WSN. In Fuzzy Systems and Knowledge Discovery (FSKD), 2012 9th International Conference on (pp. 2174-2177). IEEE, (2012, May). https://doi.org/10.1109/FSKD.2012.6233907

[13] Zhang, D., Li, G., Zheng, K., Ming, X., Pan, Z. $H$.: An energy-balanced routing method based on forward-aware factor for wireless sensor networks. IEEE transactions on industrial informatics, 10(1), 766-773. (2014). https://doi.org/10.1109/TII.2013.2250910

[14] Geetha, V. A., Kallapur, P. V., Tellajeera, S.: Clustering in wireless sensor networks: performance comparison of LEACH \& LEACHC protocols using NS2. Procedia Technology, 4, 163-170. (2012). https://doi.org/10.1016/j.protcy.2012.05.024

[15] Gambhir, S., Fatima, N.: Op-LEACH: an optimized LEACH method for busty traffic in WSNs. In 2014 Fourth International Conference on Advanced Computing \& Communication Technologies (pp. 222-229). IEEE. (2014, February). https://doi.org/10.1109/ACCT.2014.94

[16] Ankit Thakkar, "DEAL: Distance and Energy Based Advanced LEACH Protocol", Information and Communication Technology for Intelligent Systems, 2017. 